Nguyen Thi Thu Trang*, Tran Thi Mai, Nguyen Vu Giang, Tran Huu Trung, Do Van Cong, Nguyen Thuy Chinh, Trinh Hoang Trung, Tran Dai Lam and Thai Hoang*

\title{
Study on characteristics, properties, and morphology of poly(lactic acid)/chitosan/ hydroquinine green nanoparticles
}

https://doi.org/10.1515/gps-2018-0025

Received February 1, 2018; accepted July 16, 2018; previously published online August 15, 2018

\begin{abstract}
Poly(lactic acid)/chitosan (PLA/CS) green nanoparticles containing hydroquinine (Hq) were prepared by emulsion method. The content of Hq was 10-50 wt\% compared with the weight total of PLA and CS. The characteristics of these nanoparticles were analyzed by Fourier transform infrared (FTIR), differential scanning calorimetry, field emission scanning electron microscopy (FESEM), and particle size analysis. The wavenumbers of $\mathrm{C}=\mathrm{O}, \mathrm{C}=\mathrm{N}, \mathrm{OH}$, and $\mathrm{CH}_{3}$ groups in FTIR spectra of the $\mathrm{PLA} / \mathrm{CS} / \mathrm{Hq}$ (PCHq) nanoparticles shifted in comparision with neat PLA, CS, and Hq that proved the interaction between these components. The FESEM images and particle size analysis results showed that the basic particle size of PCHq nanoparticles ranged between 100 and $200 \mathrm{~nm}$. The Hq released from PLA/CS nanoparticles in $\mathrm{pH} 2$ and pH 7.4 solutions was determined by ultraviolet-visible method. The obtained results indicated that the linear regression coefficient of calibration equation of $\mathrm{Hq}$ in the above solutions approximates 1 . The Hq release from the PCHq nanoparticles includes fast release for the eight first testing hours, and then, controlled slow release. The Hq released process was obeyed according to the KorsmeyerPeppas kinetic model.
\end{abstract}

Keywords: antimalarial; chitosan (CS); drug release; hydroquinine (Hq); nanoparticles; poly(lactic acid) (PLA).

\footnotetext{
*Corresponding authors: Nguyen Thi Thu Trang and Thai Hoang, Institute for Tropical Technology, VAST, 18 Hoang Quoc Viet, Cau Giay, Hanoi, Vietnam; and Graduate University of Science and Technology, VAST, 18 Hoang Quoc Viet, Cau Giay, Hanoi, Vietnam, e-mail: ntttrang@itt.vast.vn; hoangth@itt.vast.vn Tran Thi Mai, Nguyen Vu Giang, Tran Huu Trung, Do Van Cong, Nguyen Thuy Chinh and Trinh Hoang Trung: Institute for Tropical Technology, VAST, 18 Hoang Quoc Viet, Cau Giay, Hanoi, Vietnam Tran Dai Lam: Graduate University of Science and Technology, VAST, 18 Hoang Quoc Viet, Cau Giay, Hanoi, Vietnam
}

\section{Introduction}

Malaria is known as the most common infectious disease caused by Plasmodium parasite. In 2015, risk of malaria was present in 91 countries. From 2010 to 2015, malaria incidence among populations at risk (the rate of new cases) decreased to $21 \%$ all over the world. Among all malaria-diseased age groups, about 35\% were children under 5 years [1]. There were many different drugs used for the treatment of malaria such as artemisinin, chloroquine capsules, dihydroartemisinin-piperaquin tablet combination, artesunate, quinine drugs, etc. So far, quinine is still a valuable and effective drug in the treatment of malaria. It is said to be a highly effective antimalarial drug for the treatment of malaria [2]. Quinine and its derivatives metabolize in the liver and rapidly exhaust in the urine. The half-life elimination is about $11 \mathrm{~h}$ in a healthy person, but may take longer in malaria patients. The small amounts of quinine and its derivatives excrete through bile and saliva.

Recently, the biodegradable polymers were developed for use in different fields such as agriculture, forestry, food processing, and health. Poly(lactic acid) (PLA) is the most studied because of having many properties similar to thermoplastic polymers (polyethylene, polypropylene, and polyvinyl chloride) such as high tensile strength, high module, heat resistance, etc. [3]. In addition, the PLA also has the ability of combustion resistance, anti-ultraviolet radiation [4], especially the ability of biodegradation. PLA is considered as a versatile thermoplastic polymer and is increasingly used in engineering fields [5].

Chitosan (CS), a naturally occurring polymer, has also been extensively studied due to its superior features such as nontoxic, biodegradable, high antibacterial capacity, etc. [6]. It can be obtained by deacetylation of chitin that is found in many crustaceans such as crabs, lobsters, shrimp, etc. [7].

Combining the advantages of PLA and CS, nanocomposites based on PLA and CS are being increasingly studied. Due to good adhesion, biodegradability, and biodegradability, the PLA/CS (PC) nanocomposites are 
widely applied in drug delivery, systems surgical sutures, and tissue engineering $[4,8]$.

In this work, PC nanoparticles containing antimalarial drug - hydroquinine (Hq) were prepared by the emulsion method. These nanoparticles will be expected to treat the infectious malarial disease. Thanks to the reduction of the drug use dose and the drug use time. The Fourier transform infrared spectra (FTIR), particle size distribution, morphology, thermal properties of the PLA/CS/Hq (PCHq) nanoparticles, and in vitro release of $\mathrm{Hq}$ from the nanoparticles in different $\mathrm{pH}$ solutions were reported and discussed.

\section{Materials and methods}

\subsection{Materials}

Poly(lactic acid) (density $1.25 \mathrm{~g} / \mathrm{cm}^{3}$, molecular weight $1.42 \times 10^{4} \mathrm{Da}$ ), CS (in powder, DD $>77 \%$, viscosity $1220 \mathrm{cPs}$ ), Hq (in white powder, purity $\geq 98 \%$ ) were purchased from Sigma Aldrich (USA). Dichloromethane (DCM) and acetic acid were of analytical reagent grade and used without further purification were provided by Guangdong Guanghua Chemical Factory Co. (China).

\subsection{Preparation}

An aqueous solution of the Hq drug, Hq dissolved in ethanol was poured into the PLA solution using solvent DCM to form an emulsion of water/oil. Next, the emulsion mixture of water/oil was added into $1 \%$ acetic acid solution dissolved CS and polyethylene oxide (PEO) calculated by weight. The emulsion process was carried out for $15 \mathrm{~min}$ in a MAS-II microwave machine (Sineo Microwave, China). The PCHq nanoparticles were collected by centrifugation and then washed several times with distilled water in order to remove excessive PEO emulsifier before lyophilizing using a FreeZone 2.5 equipment (Labconco, USA). In this study, the PCHq nanoparticles samples were prepared at 10, 20, 30, and $50 \mathrm{wt} \% \mathrm{Hq}$ (in comparison with PLA weight) and abbreviated as PCHq10, PCHq20, PCHq30, and PCHq50, respectively.

\subsection{Characterization}

The FTIR spectra of the PCHq nanoparticles were analyzed at room temperature by using the Nicolet/Nexus 670 spectrometer (USA). Each sample was recorded with 16 scans at a resolution of $4 \mathrm{~cm}^{-1}$.

The size distribution of the PCHq nanoparticles was measured using a Zetasizer particle size analyzer (Malvern, England).

Thermal property of the PCHq nanoparticles was analyzed by using a differential scanning calorimetry (DSC-60) thermogravimetric analyzer (Shimadzu, Japan) from room temperature to $400^{\circ} \mathrm{C}$ at a heating rate of $10^{\circ} \mathrm{C} / \mathrm{min}$ under argon atmosphere.

The morphology of the nanoparticles was observed on the FESEM images conducted using the S-4800 FESEM instrument (Hitachi, Japan). FESEM images were taken of sputtered samples with platinum coating.
The Hq released content from the nanoparticles in different $\mathrm{pH}$ solutions was calculated by UV-Vis spectroscopy method using a CINTRA 40, GBC spectrometer (USA).

\section{Results and discussion}

\subsection{FTIR spectra}

The FTIR spectra of $\mathrm{Hq}, \mathrm{PC}$, and PCHq nanoparticles are shown in Figure 1. In the FTIR spectrum of Hq, the characteristic band at $3178 \mathrm{~cm}^{-1}$ can be assigned to $-\mathrm{OH}$ bending vibration. The peaks appeared at 2929, 1622, 1510,1238 , and $1033 \mathrm{~cm}^{-1}$ corresponding to $\mathrm{CH}_{3}$ group, aryl $\mathrm{C}=\mathrm{C}$ and $\mathrm{C}=\mathrm{N}-$ conjugated group, $\mathrm{C}-\mathrm{N}$ amine group and $\mathrm{C}-\mathrm{O}-\mathrm{C}$ stretching vibration group, respectively. The FTIR spectra of $\mathrm{PCHq}$ nanoparticles indicated the characteristic peaks of the stretching vibrations of $\mathrm{C}=\mathrm{N}, \mathrm{C}-\mathrm{N}$, and $\mathrm{C}=\mathrm{C}$ groups in Hq. In addition, the shift of wavenumbers of the groups such as $\mathrm{C}=\mathrm{O}, \mathrm{C}=\mathrm{N}, \mathrm{C}-\mathrm{N}, \mathrm{C}=\mathrm{C}, \mathrm{C}-\mathrm{O}-\mathrm{C},-\mathrm{OH},-\mathrm{NH}_{2}$, and $\mathrm{COOH}$ in CS, PLA and Hq could be observed for the PCHq nanoparticles in comparison with the original PLA, CS, and Hq (Table 1). This could be explained by formation of hydrogen-bonding and dipole-dipole interactions between $\mathrm{C}=\mathrm{O}$ group in $\mathrm{PLA}$ with $\mathrm{OH}$ and $\mathrm{NH}_{2}$ groups in $\mathrm{CS}$, and $\mathrm{OH}, \mathrm{C}=\mathrm{N}$, and $\mathrm{C}-\mathrm{O}$ groups in $\mathrm{Hq}$.

\subsection{The particle size distribution}

The particle size distribution diagrams of PCHq nanoparticles using different $\mathrm{Hq}$ content $(10,20,30$, and $50 \mathrm{wt} \%$ ) are presented in Figure 2.

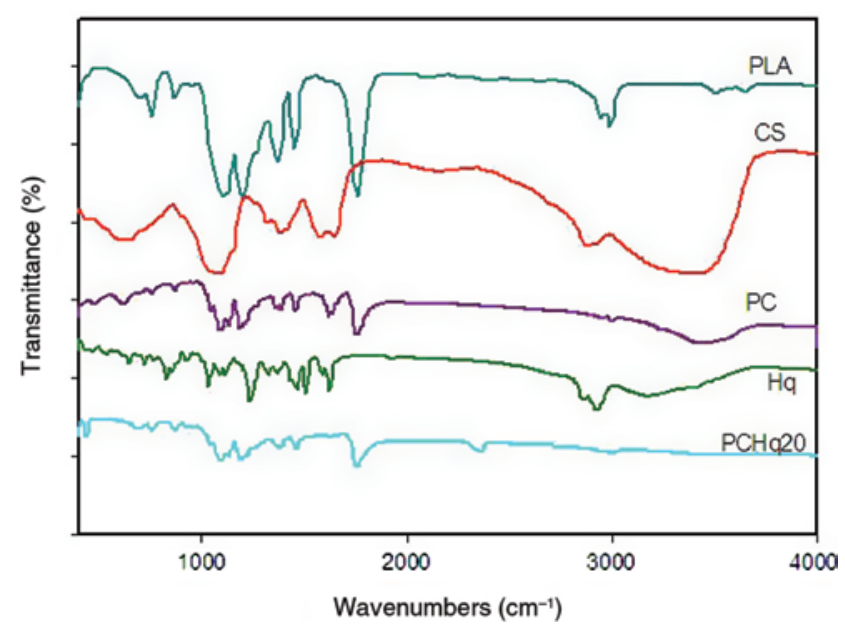

Figure 1: Fourier transform infrared spectra of hydroquinine $(\mathrm{Hq})$, poly(lactic acid)/chitosan (PLA/CS) (PC), and PLA/CS/hydroquinine (PCHq) nanoparticles. 
Table 1: Wavenumbers of characteristic groups in Fourier transform infrared spectra of hydroquinine $(\mathrm{Hq})$, and PLA/CS/hydroquinine (PCHq) nanoparticles.

\begin{tabular}{lrrrrrr}
\hline \multicolumn{6}{l}{ Wavenumbers $\left(\mathbf{c m}^{-1}\right)$} \\
\hline & Hq & PC & PCHq10 & PCHq20 & PCHq30 & PCHq50 \\
\hline$V_{\mathrm{C}=0}$ & - & 1754 & 1758 & 1759 & 1758 & 1759 \\
$\mathrm{~V}_{\mathrm{CH}}$ & 2929 & 2993 & 2997 & 2944 & 2950 & 2948 \\
$\mathrm{~V}_{\mathrm{CH}}$ & & 2939 & 2952 & 3001 & 3000 & 2999 \\
$\mathrm{~V}_{-\mathrm{OH},-\mathrm{NH}}$ & - & 3421 & 3445 & 3433 & 3507 & 3451 \\
$\mathrm{~V}_{\mathrm{C}=\mathrm{C}}$ & 1622 & - & 1625 & 1635 & 1634 & 1631 \\
$\mathrm{~V}_{\mathrm{CN}}$ & 1510 & - & 1456 & 1458 & 1458 & 1456 \\
$\mathrm{~V}_{\mathrm{C}-\mathrm{O}-\mathrm{C}}$ & & 1183 & 1189 & 1189 & 1189 & 1190 \\
& 1033 & 1084 & 1094 & 1091 & 1091 & 1096 \\
\hline
\end{tabular}

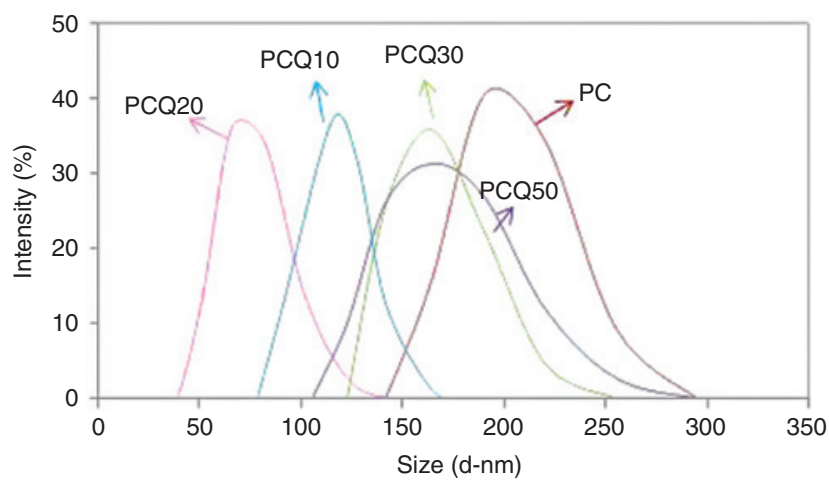

Figure 2: Particle size distribution diagrams of the $\mathrm{PCHq}$ nanoparticles using different $\mathrm{Hq}$ content.

It is clear that the particle size of PCHq nanoparticles ranged from 115 to $200 \mathrm{~nm}$. The average particle size of PCHq nanoparticles was smaller than that of the PC nanoparticles (without Hq). The change in the particle size distribution can be clarified by the hydrogen-bonding and dipole-dipole between $\mathrm{NH}_{2}$ and $\mathrm{OH}$ groups in $\mathrm{CS}$ with $\mathrm{C}=\mathrm{O}$ group in PLA, and $\mathrm{C}=\mathrm{N}, \mathrm{C}-\mathrm{O}$, and $\mathrm{OH}$ groups in Hq. This confirmed that Hq was incorporated into polymeric nanoparticles [4, 7]. The average particle size of the PCHq20 nanoparticle was smaller than that of other nanoparticles (Figure 2). This value of PCHq nanoparticles was smaller than that of PC nanoparticles loaded other drugs such as rifamicine, anthraquinone, and lamivudine $[7,9,10]$. The particle sizes of $\mathrm{PC} /$ rifamicine, $\mathrm{PC} /$ anthraquinone, and PC/lamivudine nanoparticles were 180-220, 100-200, and 300-350 nm, respectively.

\subsection{DSC analysis}

The thermal property of PCHq nanoparticles could be remarkably affected by the crystallization characteristics
Table 2: Differential scanning calorimetric data of poly(lactic acid) (PLA), chitosan (CS), and PCHq nanoparticles using different $\mathrm{Hq}$ content.

\begin{tabular}{lrrrr}
\hline Sample & $T_{\mathrm{g}}\left({ }^{\circ} \mathrm{C}\right)$ & $T_{\mathrm{m}}\left({ }^{\circ} \mathrm{C}\right)$ & $\Delta H_{\mathrm{m}}(\mathrm{J} / \mathrm{g})$ & $\boldsymbol{\chi}_{\mathrm{c}}{ }^{\mathrm{a}(\%)}$ \\
\hline PLA & 79.7 & 150.5 & 9.7 & 10.4 \\
CS & 110.7 & - & - & - \\
PCHq10 & 65.1 & 154.4 & 18.9 & 20.3 \\
PCHq20 & 68.6 & 153.2 & 22.6 & 24.7 \\
PCHq30 & 64.9 & 152.4 & 19.0 & 20.4 \\
PCHq50 & 65.2 & 154.4 & 20.4 & 21.9 \\
\hline
\end{tabular}

${ }^{a} \chi_{\mathrm{c}}(\%)=\Delta H_{\mathrm{m}} \times 100 / \Delta H_{\mathrm{m}}{ }^{*}$ where $\Delta H_{\mathrm{m}}{ }^{*}$ is the heat of fusion for completely crystallized PLA $(93.1 \mathrm{~J} / \mathrm{g}) ; T_{\mathrm{g}}$, the glass transition temperature; $T_{\mathrm{m}}$, the melting temperature; $\Delta H_{\mathrm{c}}$, the crystallization enthalpy; $\Delta H_{\mathrm{m}}$, the enthalpy of melting; $\chi_{\mathrm{c}}$, the degree of crystallinity.

of PLA and CS. The data of DSC analysis of PLA, CS, and PCHq nanoparticles using different Hq content are shown in Table 2.

From the DSC diagrams (Figure 3) and Table 2, it can be seen that neat PLA has a glass transition temperature $\left(T_{\mathrm{g}}\right)$ of $79.7^{\circ} \mathrm{C}$, and a melting temperature $\left(T_{\mathrm{m}}\right)$ of $189^{\circ} \mathrm{C}$. The $T_{\mathrm{g}}$ of CS is $110.7^{\circ} \mathrm{C}$. The PCHq nanoparticles had $T_{\mathrm{g}}$ values between the $T_{\mathrm{g}} \mathrm{S}$ of PLA and CS. The shift of $T_{\mathrm{g}}$ of PCHq nanoparticles in comparison with $T_{\mathrm{g}}$ of PLA and CS can be explained by the hydrogen-bonding and dipole-dipole interaction between $\mathrm{OH}, \mathrm{NH}_{2}, \mathrm{C}=\mathrm{O}$, and $\mathrm{C}=\mathrm{N}$ groups in CS, PLA, and $\mathrm{Hq}$ as a rearrangement of the crystal structure of PLA. This displayed the simultaneous crystallization in PCHq and nanoparticles occurred due to the interactions as aforementioned. Thus, the degree of crystallinity $\left(\chi_{c}\right)$ of the PCHq nanoparticles was higher than that of neat PLA.

\subsection{Morphology}

The FESEM images of Hq and PCHq nanoparticles using different Hq content were expressed in Figure 4. It can be seen that Hq had an amorphous form and was irregularly sized, ranging between 1 and $5 \mu \mathrm{m}$ (Figure 4A).

Figure 4(B-D) showed that the PCHq nanoparticles having a spherical shape with basic particle size was in the range $70-250 \mathrm{~nm}$. The PCHq nanoparticle using $20 \mathrm{wt} \% \mathrm{Hq}$ (PCHq20) had regular particle size and single dispersion. Its particle size was smaller than that of the nanoparticles using other Hq content (about 60-200 nm). However, all PCHq nanoparticles were agglomerated to form the particles with bigger size. The PCHq20 nanoparticle was less agglomerated than the other samples. 


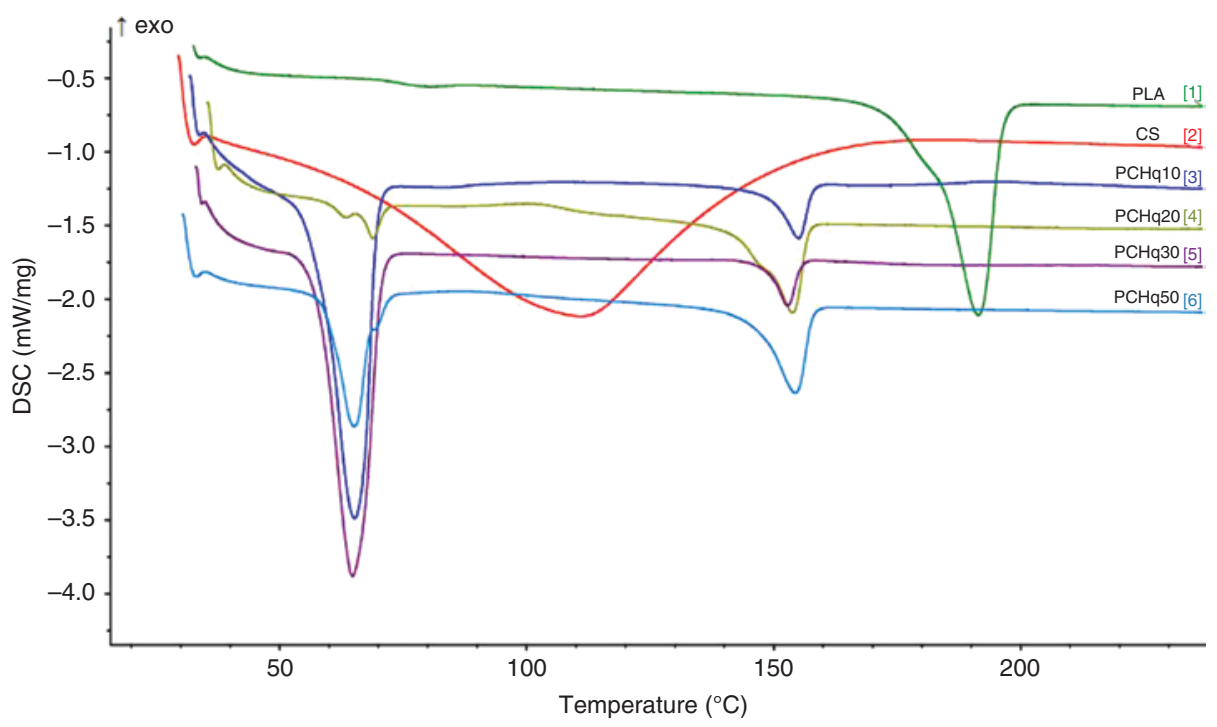

Figure 3: Differential scanning calorimetry diagrams of PLA, CS, and PCHq nanoparticles using different Hq content.
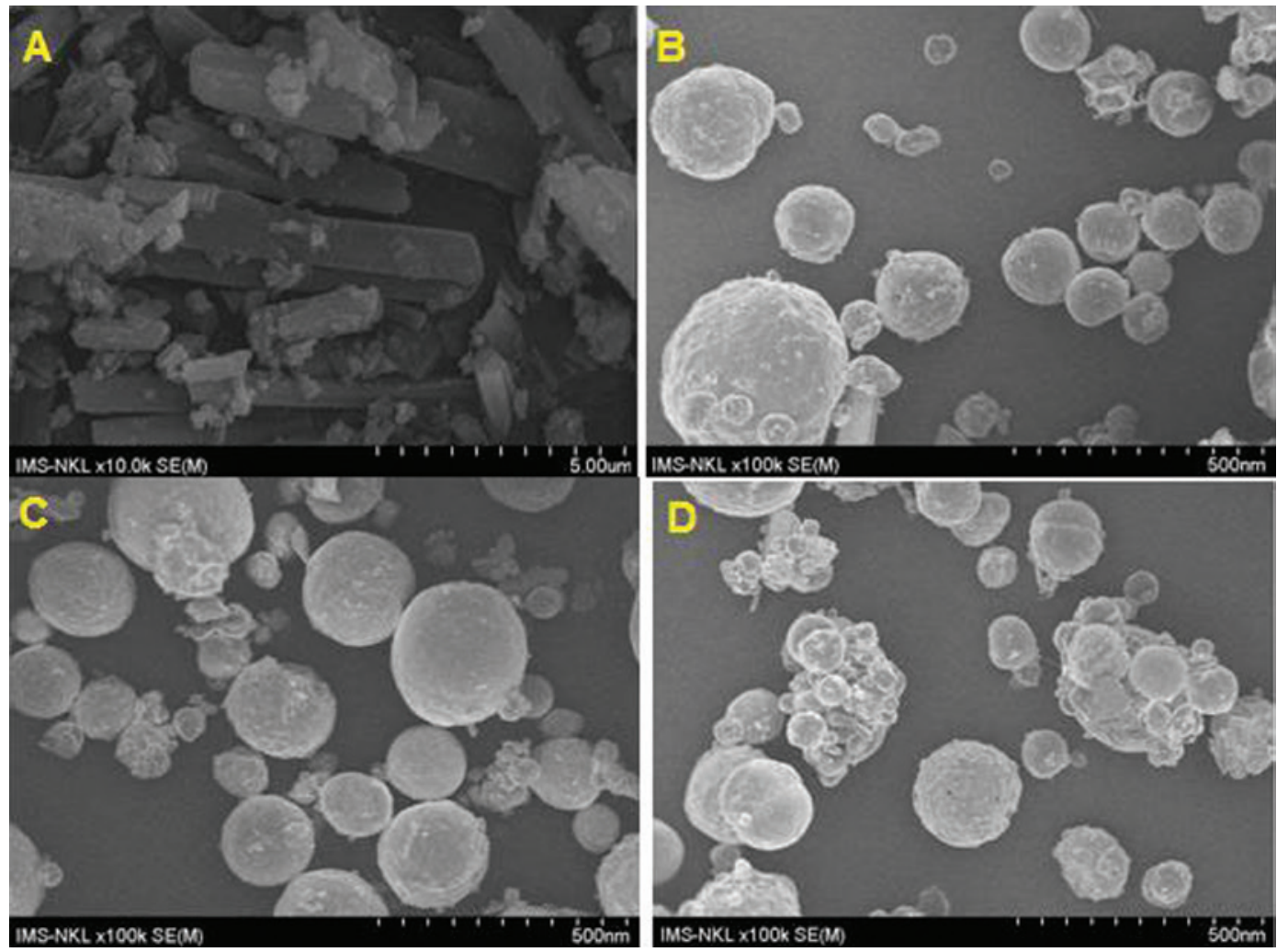

Figure 4: Field emission scanning electron microscopy images of $\mathrm{Hq}(\mathrm{A}), \mathrm{PCHq}$ nanoparticles using $20 \mathrm{wt} \% \mathrm{Hq}(\mathrm{B}), 30 \mathrm{wt} \% \mathrm{Hq}(\mathrm{C})$, and $50 \mathrm{wt} \% \mathrm{Hq}$ (D).

\subsection{In vitro drug release}

\subsubsection{Determination of $\mathrm{Hq}$ drug loading efficiency from PCHq nanoparticles}

The PCHq nanoparticles were dissolved in ethanol, then the Hq was released from the PCHq nanoparticles. The released Hq content was determined by using UV-Vis spectroscopy method. Calibration equation of Hq dissolved in ethanol: $y=6154 x+0.152$ [where $x$ is the content of $\mathrm{Hq}(\mathrm{mol} / \mathrm{l})$ and $y$ is the absorption] with linear regression coefficient $R^{2}=0.991$. The Hq released content was calculated by the following equation: $\mathrm{Hq}(\%)=m_{(t)} / m_{(0)} \times 100$ (where $m_{(t)}$ is the amount of Hq released at time $t, m_{(0)}$ is 

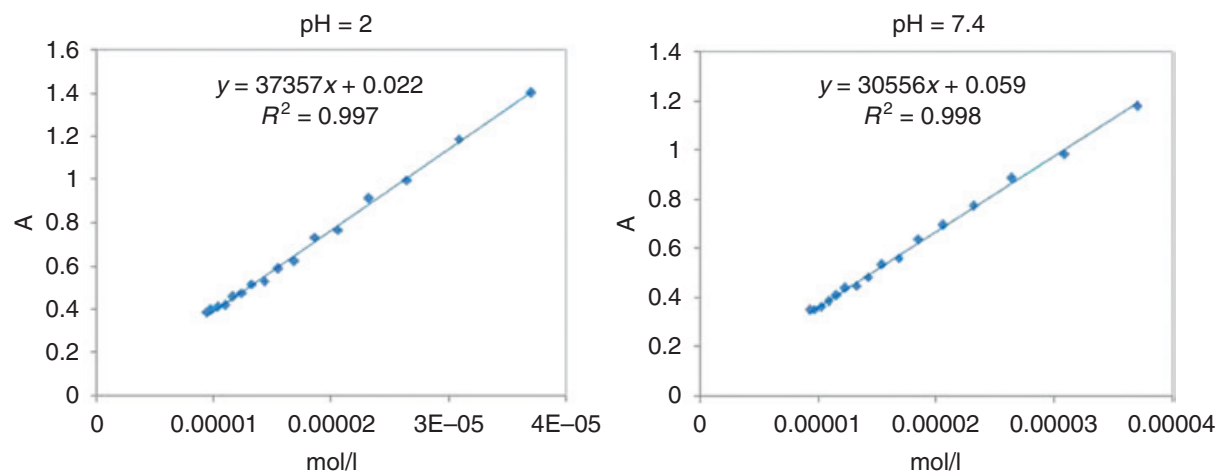

Figure 5: The absorbance versus different $\mathrm{Hq}$ content in $\mathrm{pH} 2.0$ and $\mathrm{pH} 7.4$ solutions.

the amount of initial Hq). The Hq released content from the PCHq10, PCHq20, PCHq30, and PCHq50 nanoparticles were $80.6,84.4,62.2$, and $53.4 \mathrm{wt} \%$, respectively. It is clear that the Hq released content was decreased with the rising initial Hq amout loaded to the PC nanoparticles. This can be explained by the agglomeration of Hq powder at high loaded Hq content which limit to add more Hq to the PC nanoparticles.

\subsubsection{Setting up calibration equation of $\mathrm{Hq}$ in different pH solutions}

The calibration equation of $\mathrm{Hq}$ in $\mathrm{pH} 2.0$ solution and $\mathrm{pH} 7.4$ solution were set up by using the UV-Vis spectroscopy method. Their linear regression coefficients were calculated according to the Excel software from the obtained data. The maximum wavelength of $\mathrm{Hq}$ in $\mathrm{pH} 2.0$ and $\mathrm{pH} 7.4$ solutions were 250.67 and $234.73 \mathrm{~nm}$, respectively.

The calibration equation of $\mathrm{Hq}$ in $\mathrm{pH} 2.0$ solution was $y=37357 x+0.022$ with $R^{2}=0.997$ (approximate 1) showed a linear dependence of absorbance on the Hq content at $\lambda_{\max }=250.67 \mathrm{~nm}$ in the range of $3-12 \mathrm{~g} / \mathrm{ml}$ (Figure $5 \mathrm{~A}$ ). Therefore, this wavelength was used to investigate the $\mathrm{Hq}$ content released from the PCHq nanoparticles according to testing time $(30 \mathrm{~h})$.

Similarly, the calibration equation and the regression coefficient of $\mathrm{Hq}$ in $\mathrm{pH} 7.4$ solution were displayed in Figure 5B. The calibration equation $y=30556 x+0.059$ with $R^{2}=0.998$ indicated a linear dependence of absorbance on the Hq content at $\lambda_{\max }=234.73 \mathrm{~nm}$ in the range of $3-12 \mathrm{~g} / \mathrm{ml}$.

\subsubsection{In vitro $\mathrm{Hq}$ release study}

The Hq content released from the PCHq nanoparticles using different initial Hq content in $\mathrm{pH} 2.0$ solution (corresponding to the portion of the stomach) and in $\mathrm{pH} 7.4$ solution (corresponding to the duodenum) according to testing time $(30 \mathrm{~h}$ ) were determined by the UV-Vis spectroscopy method.

\subsubsection{Effect of initial Hq content}

The Hq released content from the PCHq nanoparticles using $10-50 \mathrm{wt} \%$ (comparison with the PLA weight) in $\mathrm{pH}$ 2.0 and $\mathrm{pH} 7.4$ solutions was shown in Figure 6.
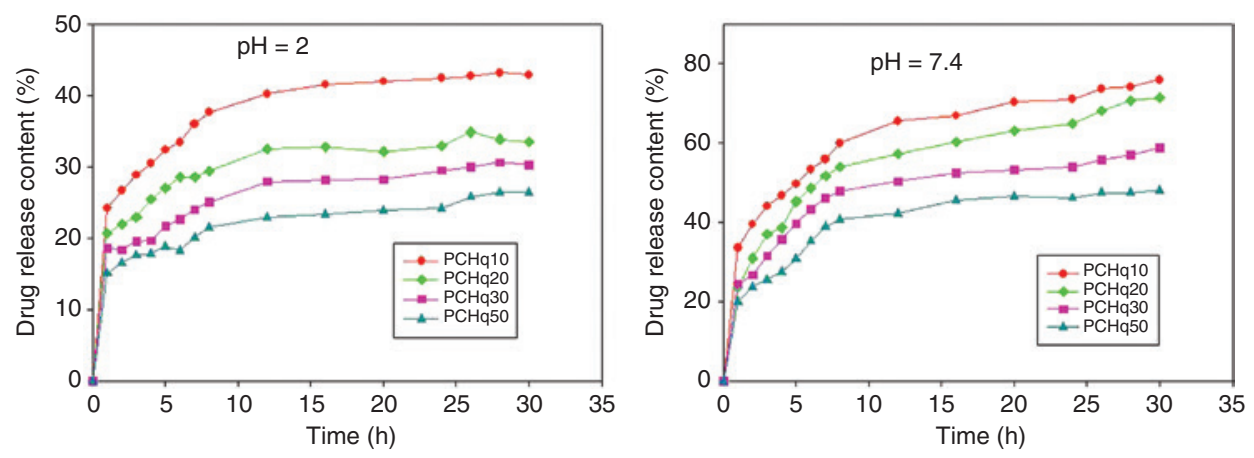

Figure 6: In vitro $\mathrm{Hq}$ released content from $\mathrm{PCHq}$ nanoparticles according to testing time. 
The Hq content released from the PCHq nanoparticles included fast released period for the first testing time and then a controlled released period (slower release). The first fast released period occurred on the surface of the samples. The slower Hq release for the second testing period was started after eight testing hours because it took time for $\mathrm{Hq}$ to diffuse through the polymer matrix. It can be seen that the Hq released content from the PCHq nanoparticles in $\mathrm{pH} 7.4$ solution was higher than that in $\mathrm{pH} 2.0$ solution. This can be explained by: in $\mathrm{pH} 2.0$ solution, the $\mathrm{Hq}$ released partially from the $\mathrm{PCHq}$ nanoparticles reacted with the acid solution to reduce the amount of $\mathrm{Hq}$ in the solution. This is consistent with the view in biomedical: $\mathrm{Hq}$ is poorly absorbed in the stomach, where its $\mathrm{pH}$ is small.

\subsubsection{Release kinetic modeling}

The HQ released kinetic study from the PCHq nanoparticles using 10-50 wt\% of initial HQ content in $\mathrm{pH} 2.0$ and $\mathrm{pH} 7.4$ solutions was determind by different models such as zero order model (ZO), first order model (FO), Higuchi model (HG), Hixson-Crowell model (HCW), and Korsmeyer-Peppas model (KMP) [11].

The Hq released process from the PCHq nanoparticles using $10-50 \mathrm{wt} \%$ of initial $\mathrm{Hq}$ content in $\mathrm{pH} 7.4$ solution was carried out for testing $30 \mathrm{~h}$ according to various kinetic models as performed in Figure 7. Figure 7(A-D) indicated that the $R^{2}$ values of $\mathrm{Hq}$ released process from the nanoparticles according to FO, HG, and HCW models were $0.941,0.901,0.966$, and 0.922 , respectively. The highest $R^{2}$ value (0.979, Table 3 ) and all $R^{2}$ values in Table 4 belonged to the KMP model which was most suitable for reflecting Hq released process from the PCHq nanoparticles in $\mathrm{pH} 7.4$ solution (Figure 7E).

The linear regression equations and the linear regression coefficient $\left(R^{2}\right)$ of Hq released from the PCHq20 nanoparticles in $\mathrm{pH} 7.4$ solution according to different kinetic models were presented in Table 3.

Similarly, the $\mathrm{Hq}$ released process from the PCHq nanoparticles using $10-50 \mathrm{wt} \%$ of initial $\mathrm{Hq}$ content in $\mathrm{pH} 2.0$ solution was carried out in $30 \mathrm{~h}$ according to various kinetic models as shown in Table 5 . The parameters of regression equations $\left(R^{2}\right.$ and $k$ ) were calculated by using the different models (ZO, FO, HG, HCW, and KMP). The highest $R^{2}$ values (0.948-0.995) corresponding to the Korsmeyer-Peppas model also expressed this model was suitable for Hq released process from the PCHq nanoparticles in $\mathrm{pH} 2.0$ solution.

Table 4 shows that the parameters of regression equation such as regression coefficient $\left(R^{2}\right)$ and constant $(k)$ that displayed the release process of $\mathrm{Hq}$ from $\mathrm{PCHq}$
A
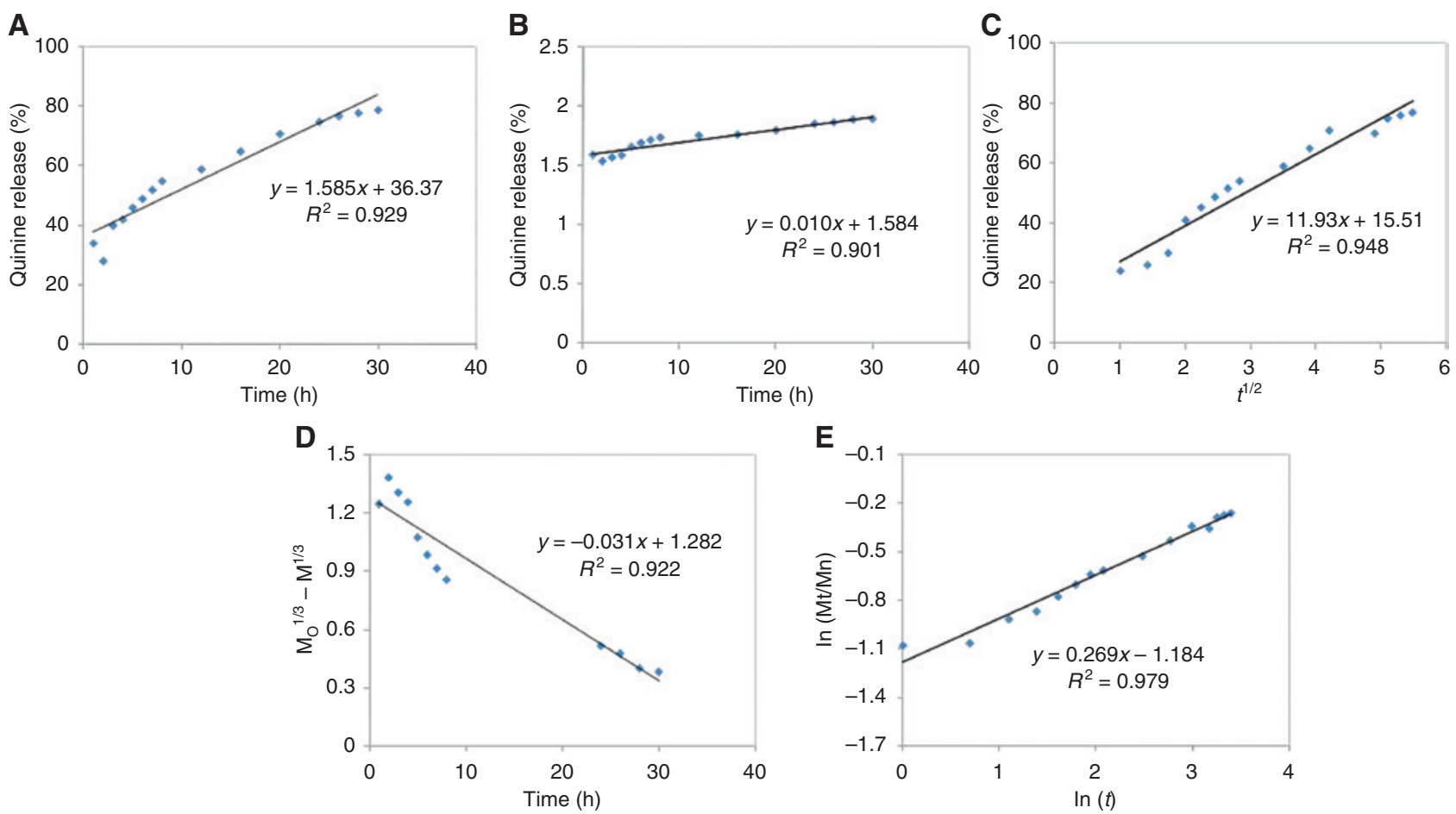

Figure 7: The Hq released kinetic from the PCHq20 nanoparticles in pH 7.4 solution [zero order model (A), first order model (B), Higuchi model (C), Hixson-Crowell model, and (D) Korsmeyer-Peppas model (E)]. 
Table 3: Regression equations and the regression coefficient $\left(R^{2}\right)$ of $\mathrm{Hq}$ released from the $\mathrm{PCHq} 20$ nanoparticles in $\mathrm{pH} 7.4$ solution according to different kinetic models.

\begin{tabular}{llr}
\hline Model & Regression equation & $\boldsymbol{R}^{2}$ \\
\hline Zero order & $y=0.557 x+28.58$ & 0.941 \\
First order & $y=0.010 x+1.584$ & 0.901 \\
Higuchi & $y=9.526+23.87$ & 0.966 \\
Hixson-Crowell & $y=-0.031 x+1.282$ & 0.922 \\
Korsmeyer-Peppas & $y=0.269 x-1.184$ & 0.979 \\
\hline
\end{tabular}

Table 4: Parameters of regression equation reflected $\mathrm{Hq}$ released process from the $\mathrm{PCHq}$ nanoparticles in $\mathrm{pH} 7.4$ solution according to different kinetic models.

\begin{tabular}{crrrr}
\hline Model & PCHq10 & PCHq20 & PCHq30 & PCHq50 \\
\hline ZO & & & & \\
$R^{2}$ & 0.895 & 0.929 & 0.948 & 0.839 \\
$k$ & 1.213 & 1.585 & 0.935 & 0.77 \\
FO & & & & \\
$R^{2}$ & 0.819 & 0.901 & 0.904 & 0.784 \\
$k$ & 0.021 & 0.010 & 0.008 & 0.009 \\
$\mathrm{HG}$ & & & & \\
$R^{2}$ & 0.964 & 0.948 & 0.979 & 0.913 \\
$k$ & 8.171 & 11.93 & 6.583 & 5.562 \\
$\mathrm{HCW}$ & & & & \\
$R^{2}$ & 0.847 & 0.922 & 0.921 & 0.803 \\
$k$ & -0.027 & -0.031 & -0.024 & -0.023 \\
$\mathrm{KMP}$ & & & & \\
$R^{2}$ & 0.984 & 0.979 & 0.974 & 0.929 \\
$k$ & 0.24 & 0.269 & 0.215 & 0.233 \\
\hline
\end{tabular}

Table 5: Parameters of regression equation reflected $\mathrm{Hq}$ released process from the $\mathrm{PCHq}$ nanoparticles in $\mathrm{pH} 2$ solution according to different kinetic models.

\begin{tabular}{crrrr}
\hline Model & PCHq10 & PCHq20 & PCHq30 & PCHq50 \\
\hline ZO & & & & \\
$R^{2}$ & 0.852 & 0.967 & 0.925 & 0.938 \\
$k$ & 0.52 & 0.375 & 0.409 & 0.345 \\
FO & & & & \\
$R^{2}$ & 0.896 & 0.968 & 0.891 & 0.905 \\
$k$ & 0.016 & 0.005 & 0.007 & 0.007 \\
HG & & & & \\
$R^{2}$ & 0.987 & 0.971 & 0.968 & 0.974 \\
$k$ & 14.0 & 10.91 & 2.902 & 2.436 \\
HCW & & & & \\
$R^{2}$ & 0.912 & 0.969 & 0.903 & 0.917 \\
$k$ & -0.018 & -0.013 & -0.016 & -0.015 \\
KMP & & & & \\
$R^{2}$ & 0.995 & 0.986 & 0.948 & 0.968 \\
$k$ & 0.177 & 0.337 & 0.173 & 0.169 \\
\hline
\end{tabular}

nanoparticles with different contents of $\mathrm{Hq}$ in $\mathrm{pH}=7.4$ are calculated based on different models (ZO, FO, HG, HCW, and KMP).

\section{Conclusions}

The FTIR spectra of Hq, PLA, CS, PC, and PCHq nanoparticles proved that $\mathrm{Hq}$ interacted with PLA, CS, and $\mathrm{Hq}$ was carried by the PC nanoparticles. The characteristic peaks of PCHq nanoparticles using different initial Hq content were shifted in comparison with the peaks of characteristic groups in original PLA, CS, and Hq. The degree of crystallinity in the PCHq nanoparticles was higher than that of neat PLA. The PCHq20 nanoparticle using $20 \mathrm{wt} \% \mathrm{Hq}$ (PCHq20) had regular particle size and single dispersion. The Hq released process from the PCHq nanoparticles included fast released period for the first testing time and then a controlled slow released period. The Korsmeyers-Peppas kinectic model was the most suitable for Hq released study in pH 7.4 and pH 2.0 solutions.

Acknowledgments: The authors would like to thank the Vietnam Academy of Science and Technology for the financial support (subject code VAST.ĐLT.05/17-18, period of 2017-2018).

\section{References}

[1] World Health Organization. Malaria, Fact sheet $N^{\circ} 94^{\prime \prime}$ (2017).

[2] Murambiwa P, Masola B, Govender T, Mukaratirwa S, Musabayane CT. Acta Tropica 2011, 118, 71-79.

[3] Gupta AP, Kumar V. Eur. Polym. J. 2007, 43, 4053-4074.

[4] Prabaharan M, Rodriguez-Perez MA, De Saja JA, Mano JF. J. Biomed. Mater. Res. B Appl. Biomater. 2007, 81, 427-434.

[5] Fronea AN, Berliozb S, Chailand JF, Panaitescu DM. Carbonhydr. Polym. 2013, 91, 377-384.

[6] Yoshihiro S, Saburo M. Biotechnol. Genet. Eng. Rev. 1995, 13, 383-420.

[7] Ashish Dev, Binulal NS, Anitha A, Nair SV, Furuike T, Tamura H, Jayakumar R. Carbohydr. Polym. 2010, 80, 833-838.

[8] Liao YZ, Xin MH, Li MC, Su S. Chinese Chem. Lett. 2007, 18, 213-216.

[9] Jeevitha D, Amarnath K. Colloids Surf. B: Biointerfaces 2013, 101, 126- 134.

[10] Rajan M, Raj V. Carbohydr. Polym. 2013, 98, 951-958.

[11] Dash S, Murthy PN, Nath L, Chowdhury P. Acta Poloniac Pharma Drug Res. 2010, 67, 217-223. 